$p=0.013)$. Unlike ocular motor changes, impairments revealed on conventional tests of cognition persisted up to 6 months.

Conclusions We found multi-domain cognitive impairments in IIH patients that were unrelated to clinical features. Marked ocular motor inhibitory control deficits were predicted by RNFL thickness but remained distinct from other cognitive changes, underscoring the significance of visual processing changes in IIH.

\section{NITROUS OXIDE INDUCED MYELO-NEUROPATHY}

${ }^{1}$ Grace Swart, ${ }^{2}$ Christopher Blair, ${ }^{3}$ Zhong X Lu, ${ }^{4}$ Solomon Yogendran, ${ }^{5}$ Joanna Offord, ${ }^{6}$ Emily Sutherland, ${ }^{7}$ Stephanie Barnes, ${ }^{6}$ Natalie Palavra, ${ }^{6}$ Phillip Cremer, ${ }^{8}$ Samuel Bolitho, ${ }^{1}$ Gabor M Halmagyi. 'Royal Prince Alfred Hospital, Camperdown, NSW, Australia; ${ }^{2}$ Liverpool Hospital, Sydney, NSW, Australia; ${ }^{3}$ Medicine, Department of Medicine, Monash University, Melbourne, VIC, Australia; ${ }^{4}$ Anaesthesia, Prince of Wales Hospital, Sydney, NSW, Australia; ${ }^{5}$ Neurology, Prince of Wales Hospital, Sydney, NSW, Australia; ${ }^{6}$ Royal North Shore Hospital, Sydney, NSW, Australia; 'Neurology, Concord Repatriation Hospital, Sydney, NSW, Australia; ${ }^{8}$ St Vincents Hospital, Sydney, NSW, Australia

\subsection{6/bmjno-2021-ANZAN.22}

Background Nitrous oxide misuse is a recognized issue worldwide. It is cheap, legal and can be bought in bulk online. Prolonged misuse inactivates vitamin B12 causing a myelo-neuropathy. Methods Review of 20 patients with nitrous-oxide induced myelo-neuropathy from tertiary hospitals between 2016-2020

Results Twenty patients had an average age of 25 years. Mean canister consumption was 150 per day for 9 months. At presentation paraesthesia and gait unsteadiness were common, and six patients were bedbound. Mean serum B12 was normal: $258 \mathrm{pmol} / \mathrm{L}(\mathrm{NR}=140-750)$ as was active B12: $94 \mathrm{pmol} / \mathrm{L}$ $(\mathrm{N}>35)$. In contrast mean serum homocysteine was high: 51 umol/L(NR=5-15). Spinal MRI( $n=19)$ showed characteristic dorsal column T2 hyperintensities. Nerve conduction studies $(n=5)$ showed a lower limb predominant axonal sensorimotor neuropathy. Patients were treated with intramuscular vitamin B12, with variable functional recovery at discharge. Three of 6 patients who were bedbound at presentation were able to walk with an aid at discharge. Of 8 patients with follow-up, most had persistent paraesthesiae and/or sensory ataxia. Admission and discharge mobility scores were not significantly correlated with serum total and active B12 levels or cumulative nitrous oxide use. However, there was an inverse trend for decreased serum active B12 level with increased cumulative nitrous oxide use (Spearman's rho -0.416, $\mathrm{p}=0.09$ ).

Conclusion Nitrous oxide misuse can cause severe but potentially reversible subacute myelo-neuropathy. Serum and active B12 can be normal, while elevated homocysteine and dorsal column high T2 signal on MRI imaging strongly support the diagnosis. Neurological deficits can improve with abstinence and B12 replacement, even in the most severely affected patients.

\section{DEVELOPMENT OF A BEDSIDE MOTION CAPTURE SYSTEM: A PILOT STUDY}

\footnotetext{
${ }^{1}$ Paul Kopanidis, ${ }^{2}$ Joe Lynch, ${ }^{3}$ Md Asikuzzaman, ${ }^{3}$ Mark Pickering, ${ }^{2}$ Diana Perriman, ${ }^{4}$ Wayne Spratford, ${ }^{1,2}$ Christian Lueck. ${ }^{1}$ Neurology, The Canberra Hospital, Garran, ACT, Australia; ${ }^{2}$ Medical School, Australian National University, Canberra, ACT, Australia; ${ }^{3}$ School of Engineering and Information Technology, The University of New South Wales, Canberra, $A C T$, Australia; ${ }^{4}$ Research Institute for Sport and Exercise, University of Canberra, Canberra, $A C T$, Australia
}

10.1136/bmjno-2021-ANZAN.23
Objectives Pronator drift is one of many clinical signs that would benefit from detailed study, but this requires accurate measurement of movement in three dimensions. The Vicon system is currently considered to be the gold standard for measurement of limb kinetics in a movement analysis lab but it cannot be used at the bedside for many reasons. This study aimed to investigate a portable camera-based motion capture system (MCS) as a clinically-useful alternative.

Methods The MCS used two commercially-available cameras arranged so as to permit stereoscopic calculation of depth (i.e. distance from the cameras), and therefore a 3-D representation of movement at the shoulder, elbow and wrist. Data were obtained simultaneously from both movement capture and Vicon systems while three normal subjects simulated four scenarios of the pronator drift test in each limb. Outputs from Vicon and MCS were analysed using Matlab to determine root mean square error (RMSE) in XYZ coordinates. A priori, an acceptable difference was considered to be an average RMSE of $<10 \mathrm{~mm}$.

Results Collectively, the studies generated 53,424 sets of data. The average RMSE in the XYZ axis was $14.9 \mathrm{~mm}$ (range 5.0$20.3 \mathrm{~mm}$ ). Inaccuracy was greatest at the wrist during trials involving larger degrees of pronation.

Conclusion The motion capture system was able to generate a 3-D trajectory of limb motion but further refinement is needed before it can be used for the purposes of clinical measurement.

\section{ANDERSEN-TAWIL SYNDROME: MULTI-SYSTEM DEEP PHENOTYPING OF A LARGE UK COHORT}

Vinojini Vivekanandam, Michael G Hanna, Emma Matthews. University College London, London, UK

\subsection{6/bmjno-2021-ANZAN.24}

Background Andersen-Tawil Syndrome (ATS) is a rare channelopathy caused by mutations in the KCNJ2 gene that encodes the ubiquitously expressed Kir2.1 potassium channel.

Methods In our paper, we describe key findings in a large UK cohort of 52 patients, pertinent to the diagnosis and management of ATS. We report a new point prevalence of 0.105 per 100000 (increased from 0.08 per 100000 ).

Results While ATS has historically been considered a triad of episodic weakness, cardiac arrhythmias and dysmorphic features, we show that there is considerable variability to this phenotype. Pure cardiac or muscle phenotypes may exist. The absence of dysmorphic features does not exclude the diagnosis. Similarly, a normal long exercise test was seen in five patients.

Importantly, we identify that the phenotype includes a significant risk of cardiac morbidity and mortality with 13\% of our cohort requiring cardiac defibrillator or pacemaker insertion and an additional 23\% reporting syncope. Syncope has been recently associated with an increased risk of life threatening arhythmic events in this cohort. Severe fixed myopathy was seen in a quarter of our cohort with $14 \%$ requiring a wheelchair or gait aid.

Conclusions This is the largest multi-system study in ATS and provides key clinical insights to improve diagnosis, as well as management recommendations to address the potential for severe muscle weakness and cardiac morbidity and mortality. 